\title{
Autoeficácia e desempenho escolar de alunos do ensino fundamental
}

\author{
Juliana da Silva \\ Universidade do Estado de Santa Catarina - SC \\ Thais Silva Beltrame \\ Universidade do Estado de Santa Catarina - SC \\ Maick da Silveira Viana \\ Universidade do Estado de Santa Catarina - SC \\ Renata Capistrano \\ Universidade do Estado de Santa Catarina - SC \\ Annelise do Vale Pereira de Oliveira \\ Universidade do Estado de Santa Catarina - SC
}

\begin{abstract}
Resumo
O estudo objetivou investigar a associação do senso de autoeficácia com o desempenho escolar, as dificuldades de aprendizagem, o sexo e a idade de crianças das séries iniciais do Ensino Fundamental. Participaram 406 escolares (56,9\% meninas), com idades entre sete e dez anos, matriculados em uma escola da região metropolitana de Florianópolis/SC. Os instrumentos utilizados foram o Teste de Desempenho Escolar (TDE) e o Roteiro de Avaliação do Senso de Autoeficácia (RASAE). Foi identificada associação significativa entre autoeficácia e desempenho escolar para todos os níveis de habilidades escolares, e aqueles com melhor desempenho apresentavam maior autoeficácia ( $p<0,001)$. Quanto ao sexo, as meninas mostraram-se mais autoeficazes do que os meninos ( $p<0,05)$. Crianças de diferentes grupos etários $(7$ a 8 e 9 a 10 anos) tiveram níveis semelhantes de autoeficácia. Sugere-se que ações com vista ao aumento da autoeficácia sejam direcionadas especialmente aos meninos e grupos com baixo desempenho, de forma a repercutirem na motivação e aprendizagem escolar.
\end{abstract}

Palavras-chave: Autoeficácia; rendimento escolar; crianças.

\section{Self-efficacy and school performance of elementary school students}

\begin{abstract}
In this study we aim at investigating the association of the feeling of self-efficacy with academic performance, learning difficulties, gender and age of children in the early grades of elementary school. The sample was composed of 406 children (56\% girls), aged 7 to 10 years, enrolled in a school in the metropolitan area of Florianópolis/SC. The instruments used were the Academic Performance Test (TDE) and Sense of selfefficacy Assessment Guide (RASAE). Statistically significant association was found between self-efficacy and academic performance for all levels of school skills and those with the best performance had higher self-efficacy $(p<0.001)$. Regarding the results between gender, girls were more self-efficacy than boys $(p<0,05)$. Children of different age groups $(7-8$ and 9-10 years) had similar levels of self-efficacy. The results suggest that actions which target the increase of self-efficacy are directed especially to boys and groups with low academic performance. This produces impacts on the motivation and school learning.
\end{abstract}

Keywords: Self-efficacy; academic achievement; children.

\section{Autoeficacia y desempeño escolar de alumnos de la enseñanza fundamental}

\section{Resumen}

El estudio tuvo por objetivo investigar la asociación del censo de autoeficacia con el desempeño escolar, las dificultades de aprendizaje, el sexo y la edad de niños de las series iniciales de la Enseñanza Fundamental. Participaron 406 escolares (el 56,9\% de muchachas), con edades entre siete y diez años, inscriptos en una escuela de la región metropolitana de Florianópolis/SC. Los instrumentos utilizados fueron el test de Desempeño Escolar (TDE) y el Rutero de Evaluación del Censo de Autoeficacia (RASAE). Se identificó asociación significativa entre autoeficacia y desempeño escolar para todos los niveles de habilidades escolares, y aquellos con mejor desempeño presentaban mayor autoeficacia ( $p<0,001$ ). Respeto al sexo, las muchachas se mostraron más auto eficaces de que los muchachos $(p<0,05)$. Niños de distintos grupos eterios $(7$ a 8 y 9 a 10 años) tuvieron niveles semejantes de autoeficacia. Se sugiere que acciones con vista al aumento de la autoeficacia sean direccionadas especialmente a los muchachos y grupos con bajo desempeño, de forma a repercutir en la motivación y aprendizaje escolar.

Palabras-clave: autoeficacia; desempeño académico; niño. 


\section{Introdução}

A Teoria Social Cognitiva (Bandura, 1986) sugere que a realização humana depende da interação entre os comportamentos pessoais e as condições ambientais. Entre os fatores que compõem a Teoria Social Cognitiva, a autoeficácia se destaca como a representante intrapessoal que mais influencia o comportamento humano (Bandura, 1977). A autoeficácia é definida como um julgamento pessoal das próprias capacidades de executar cursos de ação exigidos para se atingir certo grau de desempenho em uma determinada tarefa ou situação (Bandura, 1993). De acordo com Neves e Faria (2007), sua formação é circunstancial e contextualizada, remetendo-se para as especificidades de cada domínio particular de realização, de cada situação e até mesmo de cada tarefa.

De acordo com Costa e Boruchovich (2006), os estudos conduzidos por Bandura verificaram que a autoeficácia possui função reguladora sobre o comportamento, atuando como uma mediadora entre cognição, emoção e motivação. Tais crenças podem influenciar as aspirações e o envolvimento com metas estabelecidas, o nível de motivação, a perseverança diante das dificuldades, a resiliência às adversidades, a qualidade de pensamento analítico, a atribuição causal para o sucesso ou fracasso e outros aspectos (Medeiros, Loureiro, Linhares, \& Marturano, 2000).

O senso de autoeficácia é um tema investigado em diversos campos da Psicologia, nos quais os pesquisadores têm verificado associação com variáveis como o estresse, vícios, controle da dor, fobias, relações entre pais e filhos com deficiência e desempenho atlético (Farkas, \& Valdéz, 2010; Salvetti, \& Pimenta, 2007; Cabrera, Anzano, Sánchez, \& Méndez, 2011; Schmidt, \& Bosa, 2007; Leite, Drachler, Centeno, Pinheiro, \& Silveira, 2002; Hasting, \& Symes, 2002; Keefe, \& cols. 1997). O tema também tem recebido importante atenção no campo da pesquisa educacional (Souza, \& Brito, 2008; Oliveira, \& Soares, 2011; Rodrigues, \& Barrera, 2007; Neves, \& Faria, 2007; Pietsch, Walker, \& Chapman, 2003; Cecconello, \& Koller, 2000), inclusive em estudos envolvendo crianças com dificuldades de aprendizagem geral (Medeiros, \& cols., 2000, Loureiro, \& Medeiros, 2004; Medeiros, Loureiro, Linhares, \& Marturano, 2003) e específicas: leitura, escrita e aritmética (Souza, \& Brito, 2008, Neves, \& Faria, 2007) e com problemas afetivos ou comportamentais (Medeiros, \& cols., 2000; Galla, \& Wood, 2012; Yazici, Seyis, \& Altum, 2011). Na área escolar, as crenças de autoeficácia são convicções pessoais sobre as capacidades relacionadas às demandas específicas do cotidiano e atividades escolares (Bandura, \& Schunk, 1981).

Ao longo do Ensino Fundamental as crianças vivenciam experiências escolares que influenciam seu desempenho enquanto alunos (Oliveira, \& Soares, 2011; Molina, \& Del Prette, 2007). A autoeficácia, juntamente com outras crenças e atitudes para a aprendizagem, além de influenciar o desempenho acadêmico, também é influenciada por este (Loureiro, \& Medeiros, 2004). No caso das crianças que apresentam mau desempenho acadêmico, a baixa autoe- ficácia enfraquece a crença no próprio potencial, podendo agravar seu desempenho escolar e, por consequência, sua autoeficácia é novamente afetada por meio de um círculo vicioso (Loureiro, \& Medeiros, 2004; Medeiros, \& cols., 2003; Stevanato, Loureiro, Linhares \& Marturano, 2003). Esta influência ocorre tanto por ação direta como por seu impacto nos processos de motivação, autorregulação e autopercepção, nas expectativas de resultados, nas escolhas e interesses, os quais, por sua vez, afetam o nível e o tipo de comportamento (Bandura, 1993; Schunk, \& Pajares, 2001; Schunk, 1984).

Bandura (1986) destaca que os alunos mais autoeficazes estão mais aptos a realizar tarefas com maior grau de dificuldade, bem como a atingir níveis mais elevados de desempenho. Do mesmo modo, para Schunk e Meece (2005), escolares que não têm crenças positivas a respeito de suas capacidades mostram menor persistência em atividades escolares do que aqueles que possuem um bom senso de autoeficácia.

Além do desempenho acadêmico, outros fatores podem interferir no senso de autoeficácia. Estudos mostram diferenças entre os sexos, em especial quando a autoeficácia está relacionada a outros aspectos, como desempenho acadêmico, depressão e aceitação em grupos (Pastorelli, \& cols., 2001; Jenkins, Goodness, \& Buhrmester, 2002; Vekiri, \& Chronaki, 2008). O senso de autoeficácia de crianças pode estar relacionado também à idade, pois, de acordo com Eccles, Wigfield e Schiefele (conforme citado por Costa \& Boruchovitch, 2006), crianças com menor idade tendem a apresentar um senso de autoeficácia mais elevado do que os seus pares mais velhos. Embora as variáveis sexo e idade sejam relatadas como fatores relacionados à autoeficácia de crianças, verifica-se ainda a necessidade de estudos nacionais sobre elas, buscando resultados mais conclusivos e específicos a respeito da realidade brasileira. Com base na problemática apresentada, esta pesquisa objetivou investigar a associação do senso de autoeficácia com o desempenho escolar, as dificuldades de aprendizagem, o sexo e a idade de crianças das séries iniciais do Ensino Fundamental.

\section{Método}

\section{Participantes da pesquisa}

Participaram do estudo 406 escolares com idades entre se e dez anos (sete anos: $n=51$; oito anos: $n=113$; nove anos: $n=127$; dez anos: $n=115)$, dos quais $231(56,9 \%)$ eram meninas e 175 (43,1\%), meninos. Todos estavam matriculados nas séries iniciais de uma escola de Educação Básica da região metropolitana de Florianópolis/SC, Brasil. A média de idade dos participantes foi de $8,7( \pm 1,0)$ anos e foi semelhante para cada sexo, sendo $8,7( \pm 1,0)$ anos para as meninas e $8,8( \pm 1,0)$ anos para os meninos.

A escola atendia cerca de 800 crianças matriculadas nas séries iniciais do Ensino Fundamental. Foram seleciona- 
das para o estudo as crianças a partir do segundo ano escolar e com idade entre sete anos e dez anos e onze meses. Do total de crianças matriculadas nas primeiras séries do Ensino Básico, cerca de 600 que atendiam aos critérios de inclusão receberam o Termo de Consentimento Livre e Esclarecido TCLE, o qual deveria ser assinado por um dos pais ou por seu responsável legal, para que a criança participasse da pesquisa. Houve o retorno de 410 (68\%) TCLEs devidamente assinados, dos quais, 406 compuseram a amostra.

\section{Instrumentos}

\section{Instrumento de caracterização}

Os dados pessoais dos participantes - como sexo, idade e série escolar - foram coletados previamente à avaliação do desempenho escolar e senso de autoeficácia, por meio de um questionário elaborado especificamente para o estudo.

\section{Teste de Desempenho Escolar (TDE)}

O Teste de Desempenho Escolar (TDE) foi construído para a avaliação de escolares brasileiros de $1^{\text {a }}$ a $6^{a}$ séries do Ensino Fundamental (Stein, 1994). O instrumento tem sido amplamente utilizado em estudos realizados em diferentes regiões do Brasil (Brancalhone, Williams, \& Fogo, 2004; Capellini, Tonelotto, \& Ciasca, 2004; Cia, \& Barhan, 2008; D'Avila-Bacarj, Marturano, \& Elias, 2005; Medeiros, \& cols., 2000), mostrando-se adequado para a avaliação da aprendizagem em escolares brasileiros.

Com base no resultado do TDE é possível indicar dificuldades em escrita, aritmética e leitura. Os subtestes de escrita consistem na escrita do próprio nome e de palavras apresentadas isoladamente e em frases, sob a forma de ditado. As dificuldades em aritmética são aferidas por meio de solução oral de problemas e cálculos de operações aritméticas por escrito. Por fim, a leitura é avaliada através do reconhecimento e leitura em voz alta de palavras isoladas do contexto.

O teste é único e aplicável a todas as séries, porém para cada uma delas existe um diferente padrão de comparação, de forma que quanto maior a pontuação (para cada resposta correta é atribuído o valor de 1 (um) ponto), meIhor é o desempenho no teste. Há três classificações para o desempenho escolar: superior, médio e inferior, sendo esta última um indicativo de dificuldade de aprendizagem, que pode ser específica (dificuldade de escrita, leitura ou aritmética) ou geral.

\section{Roteiro de Avaliação do Senso de Autoeficácia (RASAE)}

O Roteiro de Avaliação do Senso de Autoeficácia (RASAE) focaliza a percepção da criança sobre o seu desempenho acadêmico e sua capacidade de realização. A utilização do RASAE para a avaliação do senso de autoeficácia deu-se com base na proposta e procedimentos apresentados por Medeiros e cols. (2000).
Este instrumento foi idealizado para ser utilizado com crianças da primeira à quarta série do Ensino Fundamental, e constitui-se de 20 afirmativas gravadas em áudio. Destas, 12 são relacionadas à percepção da capacidade relacionada ao desempenho acadêmico, compreendendo seis afirmativas com significados positivos (itens $01,03,05,07,09,20$ ) e seis afirmativas com significados negativos (itens $02,12,14$, $16,18,19)$; e oito afirmativas relacionadas à percepção de desempenho acadêmico, tendo como referência a avaliação de outros ou a comparação com os pares, compreendendo quatro afirmativas com significados positivos (itens 11, $13,15,17)$ e quatro afirmativas com significados negativos (itens 04, 06, 08, 10).

Os sujeitos dispunham de duas alternativas de respostas para cada questão (sim ou não), e cada resposta recebeia pontuação de 0 (zero) ou 1 (um). Para as respostas favoráveis a um senso de autoeficácia positivo foi atribuído o valor 1 (um), que pode corresponder a "sim" ou "não", dependendo do item em questão. No conjunto dos 20 itens, nas dez afirmativas positivas a resposta "sim" é pontuada com valor 1 (um), e nas dez afirmativas negativas a resposta é pontuada com valor 0 (zero). Por exemplo: "Na escola eu tenho me saído bem" ( $\operatorname{Sim=} 1$ e Não= 0); "Eu quero parar de estudar logo (Sim= 0 e Não= um). A somatória do total de pontos obtidos no conjunto de 20 itens corresponde ao escore total do senso de autoeficácia, de modo que quanto maior a pontuação, mais alto é o senso de autoeficácia da criança. No presente estudo, a análise da consistência interna - mensurada por meio do teste Kuder-Richardosn 20 - foi considerada adequada $(K R-20=0,790)$. A literatura apresenta valores excelentes para a consistência interna do instrumento, como podemos ver em Cruvinel e Boruchovitch (2009), com valor de 0,89.

\section{Procedimentos}

Previamente à realização do estudo houve a submissão do projeto de pesquisa ao Comitê de Ética em Pesquisa em Seres Humanos - CEP da Universidade do Estado de Santa Catarina - UDESC, o qual aprovou todos os procedimentos preestabelecidos para a realização da pesquisa. As coletas de dados foram realizadas na própria escola, nos períodos matutino e vespertino, durante o horário de aula dos participantes, com o consentimento dos pais, dos professores e da direção da escola.

Primeiramente os participantes responderam ao questionário de caracterização, o qual foi preenchido pelo pesquisador por meio de entrevista individual. Em seguida, aplicou-se o TDE em duas etapas, sendo a primeira de forma coletiva, com os testes de escrita e aritmética, e a segunda de forma individual, o teste de leitura. Posteriormente os participantes responderam verbalmente às questões do RASAE, enquanto o pesquisador assinalava as suas respostas. As avaliações do desempenho escolar e do senso de autoeficácia foram realizadas em dias diferentes, por dois motivos: 1) para não fadigar a crianças, devido ao tempo 
exposto ao TDE; e 2) para evitar interferências de possíveis dificuldades vivenciadas no TDE nas respostas do teste que mensurou o senso de autoeficácia. A ordem para aplicação dos instrumentos foi realizada de acordo com o proposto por Medeiros e cols. (2003) e Medeiros e cols. (2000).

Após a finalização da pesquisa os pesquisadores retornaram à instituição de ensino e apresentaram os principais resultados para a equipe pedagógica, professores e familiares dos participantes do estudo.

\section{Análise dos dados}

A análise dos dados foi feita por meio de estatística descritiva e inferencial. Em relação à análise descritiva, foram utilizadas medidas de frequência e percentual, tendência central (médias e medianas) e dispersão (desvios-padrão e índices máximos e mínimos). Como nenhuma variável se distribuiu normalmente (Kolmogorov-Smirnov <0,05), foram utilizados testes não paramétricos para as estatísticas inferenciais. As comparações de grupos foram feitas com a utilização dos testes de Mann-Witney e Kruskal-Wallis. As associações entre variáveis categóricas foram verificadas por meio do teste Qui-Quadrado para proporções. Em todas as análises estatísticas foi estabelecido a de 0,05 como nível de significância $(p<0,05)$.

\section{Resultados}

Foram encontradas diferenças estatisticamente significativas na autoeficácia de participantes com e sem indicativo de dificuldades de aprendizagem (tabela 1).

Quanto às dificuldades de aprendizagem, houve um maior percentual de escolares com indicativo de problemas em escrita $(n=178)$, seguido de dificuldades em matemática $(n=146)$ e leitura $(n=101)$. Do total de participantes, em 127 crianças foram encontradas dificuldades gerais. Na comparação entre os sexos, as meninas apresentaram um maior percentual de casos de dificuldades em aritmética. Em contrapartida, entre os meninos houve um maior percentual de participantes com dificuldades em leitura, mas não houve associação estatística entre estas variáveis (tabela 2).

Foram verificadas diferenças significativas na autoeficácia das crianças com diferentes níveis de desempenho escolar em todas as habilidades avaliadas (escrita, aritmética e leitura) e resultado geral do TDE (tabela 3). A comparação realizada por meio do teste $U$ de Mann-Witney demonstrou que as crianças com desempenho superior em escrita, aritmética e no TDE Geral se mostraram significativamente mais autoeficazes que os grupos médio e inferior $(p<0,05)$. A autoeficácia dos escolares com desempenho inferior também foi pior quando comparada com a das crianças com desempenho médio $(p<0,05)$. Na leitura não houve diferen-

Tabela 1. Autoeficácia de crianças estudantes do ensino fundamental em função do indicativo de dificuldades de aprendizagem em escrita, aritmética, leitura e geral.

\begin{tabular}{|c|c|c|c|c|c|c|}
\hline \multirow[b]{2}{*}{$\begin{array}{l}\text { Dificuldades de } \\
\text { Aprendizagem }\end{array}$} & \multicolumn{5}{|c|}{ Senso de Autoeficácia } & \multirow[b]{2}{*}{$\mathbf{p}$} \\
\hline & Média & Mediana & \pm & Máx. & Mín. & \\
\hline \multicolumn{7}{|l|}{ Escrita } \\
\hline Sim & 15,57 & 16,00 & 3,87 & 20,00 & 4,00 & \multirow{2}{*}{$p<0,001$} \\
\hline Não & 17,47 & 18,00 & 2,88 & 20,00 & 4,00 & \\
\hline \multicolumn{7}{|l|}{ Aritmética } \\
\hline Sim & 15,45 & 17,00 & 4,07 & 20,00 & 4,00 & \multirow{2}{*}{$p<0,001$} \\
\hline Não & 17,30 & 18,00 & 2,90 & 20,00 & 6,00 & \\
\hline \multicolumn{7}{|l|}{ Leitura } \\
\hline Sim & 15,24 & 16,00 & 4,12 & 20,00 & 4,00 & \multirow{2}{*}{$p<0,001$} \\
\hline Não & 17,10 & 18,00 & 3,11 & 20,00 & 4,00 & \\
\hline \multicolumn{7}{|l|}{ Geral } \\
\hline Sim & 15,02 & 16,00 & 4,01 & 20,00 & 4,00 & \multirow{2}{*}{$p<0,001$} \\
\hline Não & 17,37 & 18,00 & 2,93 & 20,00 & 4,00 & \\
\hline
\end{tabular}


Tabela 2. Associação entre Indicativo de dificuldades de aprendizagem em escrita, aritmética, leitura e geral e sexo de crianças estudantes do ensino fundamental $[\%(n)]$.

\begin{tabular}{lcccccccc}
\hline & \multicolumn{2}{c}{$\begin{array}{c}\text { Dificuldades em } \\
\text { Escrita }\end{array}$} & \multicolumn{2}{c}{$\begin{array}{c}\text { Dificuldade em } \\
\text { Aritmética }\end{array}$} & \multicolumn{2}{c}{$\begin{array}{c}\text { Dificuldades em } \\
\text { Leitura }\end{array}$} & $\begin{array}{c}\text { Dificuldades de } \\
\text { Aprendizagem Geral }\end{array}$ \\
\hline Sim & Não & Sim & Não & Sim & Não & Sim & Não \\
\hline Meninos & $44(77)$ & $56(98)$ & $34,3(60)$ & $65,7(115)$ & $25,7(45)$ & $74,3(130)$ & $36(63)$ & $64(112)$ \\
Meninas & $43,7(101)$ & $56,3(130)$ & $37,2(86)$ & $62,8(145)$ & $24,2(56)$ & $75,8(175)$ & $27,7(64)$ & $72,3(167)$ \\
$X^{2}$ & $0,003(p>0,05)$ & $0,375(p>0,05)$ & $0,115(p>0,05)$ & $3,187(p>0,05)$ \\
\hline
\end{tabular}

Tabela 3. Autoeficácia de crianças estudantes do ensino fundamental em função do desempenho em teste de escrita, aritmética, leitura e geral.

\begin{tabular}{|c|c|c|c|c|c|c|}
\hline \multirow[b]{2}{*}{ Classificação TDE } & \multicolumn{5}{|c|}{ Senso de Autoeficácia } & \multirow[b]{2}{*}{$\mathbf{p}$} \\
\hline & Média & Mediana & \pm & Máx. & Mín. & \\
\hline \multicolumn{7}{|l|}{ Teste de Escrita } \\
\hline Inferior ${ }^{\mathrm{a}}$ & 15,57 & 6,00 & 3,87 & 20,00 & 14,00 & \\
\hline Médio ${ }^{b}$ & 17,15 & 8,00 & 3,15 & 20,00 & 4,00 & $<0,001$ \\
\hline Superior ${ }^{c}$ & 18,37 & 9,00 & 1,61 & 20,00 & 4,00 & \\
\hline \multicolumn{7}{|l|}{ Teste de Aritmética } \\
\hline Inferiora & 15,46 & 7,00 & 4,10 & 20,00 & 9,00 & \\
\hline Médio & 17,03 & 8,00 & 2,95 & 20,00 & 6,00 & $<0,001$ \\
\hline Superior $^{c}$ & 18,06 & 9,00 & 2,58 & 20,00 & 4,00 & \\
\hline \multicolumn{7}{|l|}{ Teste de Leitura } \\
\hline Inferiora & 15,23 & 6,00 & 4,14 & 20,00 & 4,00 & \\
\hline Médio $^{b}$ & 16,81 & 8,00 & 3,40 & 20,00 & 6,00 & $<0,001$ \\
\hline Superior ${ }^{b}$ & 17,33 & 8,00 & 2,81 & 4,00 & 20,00 & \\
\hline \multicolumn{7}{|c|}{ Desempenho Acadêmico Geral } \\
\hline Inferior ${ }^{\mathrm{a}}$ & 14,94 & 16,00 & 4,09 & 20,00 & 6,00 & \\
\hline Médio ${ }^{b}$ & 17,17 & 18,00 & 2,97 & 20,00 & 4,00 & $<0,001$ \\
\hline Superior $^{c}$ & 18,08 & 19,00 & 2,37 & 20,00 & 4,00 & \\
\hline
\end{tabular}

$a, b, c$ Letras diferentes indicam grupos que se diferenciaram significativamente.

ças estatisticamente significativas entre os grupos superior e médio, porém ambos se mostraram mais autoeficazes do que o grupo inferior $(p<0,05)$. A tabela 3 apresenta os valores descritivos da autoeficácia para cada grupo, de acordo com as habilidades escolares avaliadas.

Entre os grupos etários não houve diferenças significativas na autoeficácia. A análise geral por sexos, por sua vez, identificou autoeficácia superior para as meninas (meninas: $\bar{x}=17,1 / \mathrm{M}_{\mathrm{d}}=18$; meninos: $\bar{x}=16,1 ; \mathrm{M}_{\mathrm{d}}=17$ ), sendo esta diferença estatisticamente significativa $(p<0,01)$. 0 mesmo ocorreu ao analisarmos separadamente por grupos etários, tendo as meninas se mostrado mais autoeficazes do que os meninos (tabela 4). 
Tabela 4. Autoeficácia de meninos e meninas estudantes do ensino fundamental de diferentes grupos etários.

\begin{tabular}{|c|c|c|c|c|c|c|}
\hline \multirow[b]{2}{*}{ Grupo Etário } & \multicolumn{5}{|c|}{ Senso de Autoeficácia } & \multirow[b]{2}{*}{$\mathbf{U}$} \\
\hline & Média & Mediana & \pm & Máx. & Mín. & \\
\hline \multicolumn{7}{|l|}{7 e 8 anos } \\
\hline Masculino & 15,94 & 17,00 & 4,03 & 20,00 & 5,00 & \multirow{3}{*}{$\begin{array}{c}2527,50 \\
p<0,05\end{array}$} \\
\hline Feminino & 17,56 & 18,00 & 2,67 & 20,00 & 9,00 & \\
\hline Total & 16,90 & 18,00 & 3,38 & 20,00 & 5,00 & \\
\hline \multicolumn{7}{|l|}{9 e 10 anos } \\
\hline Masculino & 16,06 & 17,00 & 3,54 & 20,00 & 4,00 & \multirow{3}{*}{$\begin{array}{l}6128,00 \\
p<0,05\end{array}$} \\
\hline Feminino & 16,78 & 18,00 & 3,51 & 20,00 & 4,00 & \\
\hline Total & 16,46 & 17,00 & 3,54 & 20,00 & 4,00 & \\
\hline
\end{tabular}

\section{Discussão}

O objetivo do presente estudo foi investigar a associação do senso de autoeficácia com o desempenho escolar, as dificuldades de aprendizagem, o sexo e a idade de crianças das séries iniciais do Ensino Fundamental.

O impacto das variáveis psicológicas na aprendizagem escolar tem sido enfatizado nas últimas décadas (Zumberg, Chang, \& Sanna, 2008; Bandura, Caprara, Barbaranelli, \& Pastorelli, 2001; Schunk, \& Pajares, 2001; Bandura, 1997; Bandura, 1993). O presente estudo demonstrou que crianças com pior desempenho escolar apresentaram senso de autoeficácia também inferior. Resultados semelhantes foram encontrados por Neves e Faria (2007), as quais verificaram que alunos com melhor aproveitamento em disciplinas como Português e Matemática tendem a apresentar uma melhor autoeficácia, tanto nas disciplinas citadas como em outras disciplinas ou áreas. De acordo com a literatura, esta situação é esperada, e ainda pode ser agravada, desenvolvendo um círculo vicioso, pois a pouca crença no potencial escolar pode piorar o desempenho nas disciplinas escolares e por consequência a autoeficácia torna-se ainda mais enfraquecida (Loureiro, \& Medeiros, 2004; Medeiros, \& cols., 2003; Stevanato, \& cols., 2003). As crianças com baixo senso de autoeficácia em um dado domínio tendem a evitar tarefas difíceis, percebendo-as como uma ameaça pessoal (Costa, \& Boruchovitch, 2006). Elas apresentam baixas aspirações e pouco envolvimento com metas, tendo preocupações principalmente com o autodiagnóstico, e não em ter um melhor desempenho (Medeiros, 2000). Por outro lado, a autoeficácia correlaciona-se positivamente também com estratégias de autorregulação e negativamente com a ansiedade e o estresse (Joo, Bong, \& Choi, 2000).

Identificar os escolares com baixo senso de autoeficácia é importante, uma vez que este pode ser forte preditor de baixa performance escolar (Pietsch, Walker, \& Chapman, 2003), influenciando inclusive na escolha de uma profissão. Um dos principais fatores para tal é a compatibilidade entre as habilidades presentes no indivíduo e o que ele acredita poder desempenhar (Schunk, \& Pajares, 2001; Betz, \& Hacket, 2006). Pessoas com dificuldades de aprendizagem tendem a escolher profissões menos competitivas, pois, com base em suas experiências de frustrações passadas, não acreditam possuir capacidade para determinadas profissões consideradas de mais difícil acesso, ou que acarretem grandes responsabilidades futuras (Kerka, 2002).

Pessoas com dificuldades de aprendizagem possuem menor senso de autoeficácia escolar do que aquelas sem dificuldades (Clever, Bear, \& Juvonen, 1992; Medeiros, 2000; Medeiros, \& cols., 2003). Em um estudo semelhante ao presente, Medeiros e cols. (2000) compararam o senso de autoeficácia de escolares com e sem dificuldades de aprendizagem, sendo verificados resultados mais positivos entre os escolares sem dificuldades. Ao compararem os fatores que interferem na autoeficácia destes grupos, Hampton e Mason (2003) verificaram que as crianças com dificuldades de aprendizagem possuíam menos realizações e modelos passados (importantes fontes de autoeficácia) e altos níveis de ansiedade. Além disso, tanto os índices de autoeficácia ligados às tarefas da sala de aula quanto a organização das atividades escolares foram significativamente inferiores aos apresentados pelas crianças sem dificuldades. A literatura também indica que não apenas crianças com dificuldades de aprendizagem, mas também aquelas com baixo aproveitamento nas tarefas escolares, mostram uma autoeficácia enfraquecida e baixa autoestima, e tendem a desistir das atividades que Ihes imponham desafio (Clever, \& cols.,1992; Pietsch, Walker \& Chapman, 2003; Cairney, \& cols., 2005; Souza \& Brito, 2008, Rodrigues \& Barrera, 2007; ). 
Em relação ao sexo, verificou-se que os meninos mostraram-se menos autoeficazes do que as meninas, tanto na amostra de um modo geral como nos grupos de sete a oito e de nove a dez anos. Estudos atribuem estas diferenças a maiores dificuldades de aprendizagem escolar entre alunos do sexo masculino (Saigal, Hoult, Stoskopf, Rosenbaum, \& Streiner, 1998; Ehrenberg, 1991; Santos, \& Graminha, 2006), o que indica que este pode ser um dos fatores que contribuem para o enfraquecimento da autoeficácia dos meninos; contudo, esta explicação não é coerente com o presente estudo, pois nele não houve diferenciação entre os meninos e meninas quanto às dificuldades de aprendizagem.

Pastorelli e cols. (2001) examinaram o senso de autoeficácia de escolares com idade entre dez e quinze anos, residentes em três países (Itália, Hungria e Polônia). Apesar da utilização de uma faixa etária diferente da abrangida pelo presente estudo, em todas as amostras as meninas foram mais autoeficazes que os meninos, tanto academicamente quanto em resistir à pressão dos pares no envolvimento em atividades transgressivas (Pastorelli, \& cols., 2001). Resultados semelhantes foram encontrados por Cecconello e Koller (2000), os quais verificaram que as meninas apresentaram não só melhor autoeficácia, mas também mais competência social do que os meninos. Gehlbach e cols. (2008), por sua vez, ao pesquisarem a influência do sexo na percepção da autoeficácia, não encontraram diferenças.

Por outro lado, Schunk e Pajares (2001) e Vekiri e Chronaki (2008) apresentaram estudos que apontam uma maior autoeficácia masculina para as ciências exatas e tecnológicas. Os autores destacam ainda que durante os primeiros anos de escolaridade a tendência é que ambos os sexos apresentem valores semelhantes da autoeficácia (Schunk, \& Pajares, 2001), sendo que as diferenças surgiriam durante a transição da infância para a adolescência. Neste período a autoeficácia masculina tende a crescer, enquanto ocorre um declínio entre as meninas (Wigfield, Guthrie, Tonks, \& Perencevich, 2004). Estas mudanças estão diretamente relacionadas às personalidades típicas de meninos e meninas. Os meninos tendem a exaltar mais suas habilidades e capacidades, enquanto as meninas são mais modestas em suas realizações (Schunk, \& Pajares, 2001).

Não foram encontradas diferenças na autoeficácia de crianças dos diferentes grupos etários investigados. Embora a literatura consultada tenha trazido poucas informações a respeito deste tema, destaca-se o estudo de Cecconello e Koller (2000), no qual também não se verificaram diferenças significativas na autoeficácia de crianças de diferentes idades. Por outro lado, de acordo com Eccles e cols. (conforme citado por Costa, \& Boruchovitch, 2006) e Schunk e Pajares (2001), essa diferença é esperada, pois crianças mais jovens tendem a superestimar suas capacidades em razão da utilização de resultados imediatos e aparentes, não sendo realizada uma análise sistemática, uma vez que estas crianças ainda não possuem vivências necessárias para tal. Os autores sugerem ainda que o avanço da idade favorece uma exatidão maior na avaliação deste constructo, tornando-o fortemente relacionado com o desempenho escolar obtido.
O senso de autoeficácia começa a ser estimulado por pais e cuidadores ainda no início da primeira infância. Eles proporcionam experiências que estimulam a autoeficácia das crianças, uma vez que estas ainda não possuem conhecimentos próprios para agirem sobre os acontecimentos de seu dia a dia (Schunk, \& Pajares, 2001), os quais só adquirirão ao longo dos anos de idade escolar, juntamente com as principais informações sobre si e sobre as próprias capacidades que são fornecidas pelo ambiente da escola, no qual os professores são os mediadores para o cultivo de uma boa percepção da autoeficácia. Desta forma, os professores devem procurar motivar a turma, proporcionando experiências agradáveis e desafiadoras, de modo que os alunos possam transpor os obstáculos que Ihes são colocados (Bzuneck, 2001; Molina, \& Del Prette, 2007).

A análise da autoeficácia de escolares e de suas variáveis associadas proporciona informações a respeito de medidas que podem auxiliar no processo de escolarização de crianças brasileiras, mas o presente estudo apresenta algumas limitações, como aquelas inerentes às pesquisas transversais (Bastos, \& Duquia, 2007), a não aquisição de informações sobre o histórico escolar dos participantes e a amostragem por conveniência, a qual impossibilita a generalização dos resultados para outros contextos. Por outro lado, os resultados oferecem indícios sobre o comportamento de escolares e concorda com a maior parte dos estudos nacionais e internacionais relacionados ao tema (Clever, Bear, \& Juvonen, 1992; Medeiros, 2000; Pastorelli, \& cols. 2001; Medeiros, \& cols., 2003; Neves, \& Faria, 2007; Souza, \& Brito, 2008; Rodrigues, \& Barrera, 2007;). Isto sugere um comportamento semelhante entre as crianças catarinenses investigadas e a população de escolares em geral.

\section{Considerações finais}

Os resultados demonstraram que a autoeficácia foi mais elevada entre as crianças com melhor desempenho acadêmico, sem indicativos de dificuldade de aprendizagem e do sexo feminino. Por outro lado, não foram identificadas diferenças no senso de autoeficácia de crianças em diferentes faixas etárias. Sugere-se que aqueles grupos menos autoeficazes recebam maior atenção de suas famílias e da comunidade escolar, com intervenções que favoreçam o senso de autoeficácia, tendo em vista suas repercussões sobre a motivação e a aprendizagem.

Para futuras pesquisas desta área, sugere-se a investigação de fatores do contexto escolar, como a relação com os pares e os professores, o histórico escolar e características sociais e demográficas das crianças, tendo em vista que o controle destes fatores possibilitará conclusões mais seguras a respeito da autoeficácia infantil e das associações aqui analisadas. Sugere-se ainda a realização de estudos com amostras aleatórias representativas, bem como com delineamentos longitudinais e experimentais, os quais possibilitarão generalizações e o estabelecimento de relações mais precisas. 


\section{Referências}

Bandura, A. (1977). Self-efficacy toward a unifying theory of behavioral change. Psychological Review, 84(2), 191-215.

Bandura, A. (1986). Social foundations of thought and action: A social cognitive theory. Englewood Cliffs, NJ: Prentice- Hall.

Bandura, A. (1993). Perceived self-efficacy in cognitive development and functioning. Educational Psychologist, 28(2), 117-148.

Bandura, A. (1997). Self-efficacy: the exercise of control. Worth Publishers, New York.

Bandura, A., Caprara, V. G., Barbaranelli, C., \& Pastorelli, C. (2001). Sociocognitive and self-regulatory mechanisms governing transgressive behavior. Journal of Personality and Social Psychology, 80(1), 125-135.

Bandura, A., \& Schunk, D. H. (1981). Cultivating competence, selfefficacy, and intrinsic interest through proximal self-motivation. Journal of Personality and Social Psychology, 41(3), 586-598.

Bastos, J. L. D., \& Duquia, R. P. (2007) Um dos delineamentos mais empregados em epidemiologia: estudo transversal. Scientia Medica, 17(4), 229-232.

Betz, N., \& Hackett, G. (2006). Career self-efficacy theory: back to the future. Journal of Career Assessment, 14(1), 3-11.

Brancalhone, P. G., Fogo, J. C., \& Williams, L. C. A. (2004). Children exposed to marital violence: Assessment of academic performance. Psicologia: Teoria e Pesquisa, 20(2), 113-117.

Bzuneck, J. A. As Crenças de auto-eficácia e seu papel na motivação do aluno. Em Boruchovitch, E., \& Bzuneck, J. A. (Org.). (2001). A motivação do aluno: Contribuições da Psicologia Contemporânea (pp. 116-133). Petrópolis: Editora Vozes.

Cabrera, T. F., Anzano, S. M., Sánchez, I. M. H., Méndez, S. R., \& Olmo, A. F. (2011). Construcción y validación de una escala de autoeficacia para la actividad física. Revista Española de Salud Pública, 85(4), 405-417.

Cairney, J., Hay, J. A., Faught, B. E., Wade, T. J., Corna, L., \& Flouris, A. (2005). Developmental coordination disorder, generalized selfefficacy toward physical activity, and participation in organized and free play activities. The Journal of Pediatrics, 147(4), 515-520.

Capellini, S. A., Tonelotto, J. M. F., \& Ciasca, S. M. (2004). Medidas de desempenho escolar: Avaliação formal e opinião dos professores. Revista Estudos de Psicologia, 21(2), 79-90.

Cecconello, A. M., \& Koller, S. H. (2000). Competência social e empatia: um estudo com crianças em situação de pobreza. Estudos de Psicologia, 5(1), 71-93.
Cia, F., \& Barhan, E. J. (2008). Estabelecendo relação entre autoconceito e desempenho acadêmico de crianças escolares. Psico, 39(1), 21-27.

Clever, A., Bear, G., \& Juvomen, J. (1992). Discrepancies between competence and importance in self-perceptions of children in integrated classes. Journal of Special Education, 26(2), 125-138.

Costa, E. R., \& Boruchovitch, E. A. (2006). Auto-eficácia e a motivação para aprender: Considerações para o desempenho escolar dos alunos. Em Azzi, R. G., \& Polydoro, S. A. J. (Orgs.). Auto-eficácia em diferentes contextos. (pp. 87-109). Campinas: Editora Alínea

Cruvinel, M., \& Boruchovitch, E. (2009). Sintomas de Depressão Infantil e Ambiente Familiar. Psicologia em Pesquisa, 3(1), 87-100.

D'avila-Bacarj, K. M. G., Maturano, E. M., \& Elias, L. C. S. (2005). Suporte parental: um estudo sobre crianças com queixas escolares. Psicologia em Estudo, 10 (1), 107-115.

Ehrenberg, A. (1991). Le culte de la performance. Paris: CalmannLévy.

Farkas, C., \& Valdés, N. (2010). Maternal stress and perceptions of self-efficacy in socioeconomically disadvantaged mothers: An explicative model. Infant Behavior and Development, 33(4), 654662.

Galla, B. M., \& Wood, J. J. (2012). Emotional self-efficacy moderates anxiety-related impairments in math performance in elementary school-age youth. Personality and Individual Differences, 52(2), 118-122.

Gehlbach, H., Brown, S. W., loannou, A., Boyer, M. A., Hudson, N., Solomon, A. N., Maneggia, D., \& Janik, L. (2008). Increasing interest in social studies: Social perspective taking and self-efficacy in stimulating simulations. Contemporary Educational Psychology, 33(4), 894-914.

Hampton, N. Z., \& Mason, E. (2003). Learning disabilities, gender, sources of efficacy,self-efficacy beliefs, and academic achievement. Journal of School Psychology, 41(2), 101-112.

Hasting, R. P., \& Symes, M. D. (2002). Early intensive behavioral intervation for chindren with autism: parental therapeutic selfefficacy. Research in Developmental Disabilities, 23(5), 332-341.

Jenkins, R. S., Goodness, K. \& Buhrmester, D. (2002). Gender differences in early adolescents' relationship qualities, self-efficacy, and depression symptoms. Journal of Early Adolescence, 22(3), 277-309.

Joo, Y. J., Bong, M. \& Choi, H. J. (2000). Self-efficacy for self-regulated learning, academic self-efficacy, and internet self-efficacy in webbased instruction. ETR\&D, 48(2), 5-17. 
Keefe, F. J., Kashikar, Z. S., Robinson, E., Salley, A., Beaupre, P., Caldwell, D., Baucom, D. \& Haythornthwaite, J. (1997). Pain coping strategies that predict patients and spouses ratings of patients selfefficacy. Pain, 73(2), 191-199.

Kerka, S. (2002). Learning Disabilities and Carrer Development. Practice Application Brief, 20, 2-5.

Leite, J. C. C., Drachler, M. L., Centeno, M. O., Pinheiro, C. A. T., \& Silveira, V. L. (2002). Desenvolvimento de uma escala de autoeficácia para adesão ao tratamento anti-retroviral. Psicologia: Reflexão e Crítica, 15(1), 121-133.

Loureiro, S. R., \& Medeiros, P. C. O. (2004). Senso de auto-eficácia de crianças com dificuldades de aprendizagem. Em Maturano, E. M. Vulnerabilidade e proteção. São Paulo: Casa do psicólogo.

Medeiros, P. C. (2000). Crianças com dificuldades de aprendizagem: Avaliação do senso de auto-eficácia e dos aspectos comportamentais. Dissertação de Mestrado em Ciências, Universidade de São Paulo, São Paulo.

Medeiros, P. C., Loureiro, S. R., Linhares, M. B. M., \& Marturano, E. M. (2000). A autoeficácia e os aspectos comportamentais de crianças com dificuldade de aprendizagem. Psicologia Reflexão e Crítica, 13(3), 327-336.

Medeiros, P. C., Loureiro, S. R., Linhares, M. B. B., \& Marturano, E. M. (2003). O senso de auto-eficácia e o comportamento orientado para a aprendizagem em crianças com queixas de dificuldades de aprendizagem. Estudos de Psicologia, 8(1), 93-105.

Molina, R. C. M., \& Del Prette, A. (2007) Mudanças no status sociométrico negativo de alunos com dificuldades de aprendizagem. Psicologia Escolar e Educacional, 11(2), 299-310.

Neves, S. P., \& Faria, L. (2007). Auto-eficácia acadêmica e atribuições causais em português e matemáticas. Análise Psicológica, 4(25), 635-652.

Oliveira, M. B., \& Soares, A. B. (2011). Auto-eficácia, raciocínio verbal e desempenho escolar em estudantes. Psicologia: Teoria e Pesquisa, 27(1), 33-39.

Pastorelli, C., Caprara, G. V., Barbaranelli, C., Rola, J., Rozsa, S., \& Bandura, A. (2001). The structure of children's perceived self-efficacy: A Cross-National Study. European Journal of Psychological Assessment, 17(2), 87-97.

Pietsch, J., Walker, R., \& Chapman, E. (2003). The relationship among self-concept, self-efficacy, and performance in mathematics during secondary school. Journal of Educational Psychology, 95(3), 589603 .

Rodrigues, L. C., \& Barrera, S. D. (2007) Auto-eficácia e desempenho escolar em alunos do Ensino Fundamental. Psicologia em Pesquisa, 1(2), 41-53.
Saigal, S., Hoult, L. A., Stoskopf, B. L., Rosenbaum, P. L., \& Streiner, D. L. (1998). Role of gender and birthweight in the stability of psychometric scores and the incidence of specific learning disabilities (Id) among "normal" elbw and term children. International Pediatrics Research Foundation, 43(2), 325-331.

Salvetti, M. G., \& Pimenta, C. A. M. (2007). Dor crônica e a crença de auto-eficácia. Revista Escola da Enfermagem, 41(1), 135-140.

Santos, P. L., \& Graminha, S. S. V. (2006) Problemas Emocionais e Comportamentais Associados ao Baixo Rendimento Acadêmico. Estudos de Psicologia, 11(1), 101-109.

Schmidt, C., \& Bosa, C. (2007). Estresse e auto-eficácia de mães de pessoas com autismo. Arquivos Brasileiros de Psicologia, 59(2), 179-191.

Schunk, D. H. (1984). Self-efficacy perspective on achievement behavior. Educational Psychologist, 19(1), 48-58.

Schunk, D. H., \& Meece, J. L. (2005). Self-efficacy development in adolecents. Em Urdan, T., \& Pajares, F. Self-efficacy beliefs of adolescents (pp. 71-96). Charlotte: Information Age Publishing.

Schunk, D. H., \& Pajares, F. (2001). The development of academic self-efficacy. Em Wigfield, A., \& Eccles, J. (Orgs.). Development of Achievement Motivation (pp. 1-27). San Diego: Academic Pres.

Souza, L. F. N. I., \& Brito, M. R. F. (2008). Crenças de autoeficácia, autoconceito e desempenho em matemática. Estudos de Psicologia, 25(2), 193-201.

Stein, L. M. (1994). Teste de Desempenho Escolar - TDE. São Paulo: Casa do Psicólogo.

Stevanato, I. S., Loureiro, S. R., Linhares, M. B. M., \& Marturano, E. M. (2003). Auto-conceito de crianças com dificuldades de aprendizagem e problemas de comportamento. Psicologia em Estudo, 1(8), 67-76.

Vekiri, I., \& Chronaki, A. (2008). Gender issues in technology use: perceived social support, computer self-efficacy and value beliefs, and computer use beyond school. Computers \& Education, 51(3), $1392-1404$.

Zumberg, K. M., Chang, E. C., \& Sanna, L. J. (2008). Does problem orientation involve more than generalized self-efficacy? Predicting psychological and physical functioning in college students. Personality and Individual Differences, 45, 328-332.

Wigfield, A., Guthrie, J. T., Tonks, S., \& Perencevich, K. C. (2004). Children's motivation for reading: Domain specificity and instructional influences. The Journal of Educational Research, 97(6), 299-303.

Yazici, H., Seyis, S., \& Altun, F. (2011). Emotional intelligence and self-efficacy beliefs as predictors of academic achievement among 
high school students. Procedia Social and Behavioral Sciences,

15, 2319-2323.

Recebido em: 08/03/2013

1a. Reformulação em: 22/10/2013

2a. Reformulação: 27/11/2013

Aprovado em: 04/12/2013

\section{Sobre os autores}

Juliana da Silva (julianaef@gmail.com)

Doutorado em Ciências do Movimento Humano - Universidade do Estado de Santa Catarina - UDESC, mestre em Ciências do Movimento Humano

Thais Silva Beltrame (tsbeltrame@gmail.com)

Departamento de Ciências da Saúde - Universidade do Estado de Santa Catarina, Doutora em Ciências do Movimento Humano.

Maick da Silveira Viana (efísica@gmail.com)

Doutorado em Ciências do Movimento Humano - Universidade do Estado de Santa Catarina - UDESC, mestre em Ciências do Movimento Humano.

Renata Capistrano (recapis@gmail.com)

Mestrado em Ciências do Movimento Humano - Universidade do Estado de Santa Catarina - UDESC, graduada em Educação Física.

Annelise do Vale Pereira de Oliveira (annedovale@gmail.com)

Mestrado em Ciências do Movimento Humano - Universidade do Estado de Santa Catarina - UDESC, graduada em Educação Física.

Fonte financiadora: Este trabalho recebeu apoio financeiro PROBIC/UDESC (Programa de Bolsa de Iniciação Científica) e bolsa DS-CAPES de mestrado (Coordenação de Aperfeiçoamento de Pessoal de Nível Superior).

Trabalho derivado de uma dissertação de mestrado 